\title{
Oligophrenin-1: the link between calcium-regulated exocytosis and compensatory endocytosis in neuroendocrine cells
}

\author{
Catherine Estay-Ahumada ${ }^{1}$, Stéphane Ory ${ }^{1}$, Stéphane Gasman ${ }^{1}$, Sébastien Houy ${ }^{1,2}$ \\ ${ }^{1}$ Institut des Neurosciences Cellulaires et Intégratives (INCI), Centre National de la Recherche Scientifique (CNRS UPR 3212) \& \\ Université de Strasbourg, 5 rue Blaise Pascal, 67084 Strasbourg, France \\ ${ }^{2}$ Department of Neuroscience and Pharmacology, Faculty of Health and Medical Sciences, University of Copenhagen, 2200N \\ Copenhagen, Denmark
}

Correspondence: Sébastien Houy

E-mail: shouy@sund.ku.dk

Received: March 09, 2016

Published online: April 12, 2016

\begin{abstract}
In neuroendocrine cells, hormones and neuropeptides are released from large-dense core vesicles (secretory granules) by calcium-regulated exocytosis. Following exocytosis, compensatory uptake of membrane is required to maintain membrane homeostasis and allow recycling of secretory vesicle membranes. How these cells initiate and regulate this compensatory endocytosis remains poorly understood. Our recent data suggests that oligophrenin-1 (OPHN1) is a link coupling calcium-regulated exocytosis to compensatory endocytosis of secretory granules in the adrenal chromaffin cells (Houy et al., 2015, J Neurosci. 2015, 35:11045-55). Here, we highlight the major evidence and discuss how OPHN1 could couple these two processes.
\end{abstract}

Keywords: calcium-regulated exocytosis; compensatory endocytosis; neuroendocrine secretion; chromaffin cells, oligophrenin1; Rho-GTPases; amperometry

To cite this article: Catherine Estay-Ahumada, et al. Oligophrenin-1: the link between calcium-regulated exocytosis and compensatory endocytosis in neuroendocrine cells. Neurosci Commun 2016; 2: e1251. doi: 10.14800/nc.1251.

Copyright: () 2016 The Authors. Licensed under a Creative Commons Attribution 4.0 International License which allows users including authors of articles to copy and redistribute the material in any medium or format, in addition to remix, transform, and build upon the material for any purpose, even commercially, as long as the author and original source are properly cited or credited.

\section{Introduction}

Intracellular membrane trafficking along endocytotic and secretory pathways plays a critical role in diverse cellular functions including developmental and pathological processes. Proteins and lipids destined for transport to distinct locations are collectively assembled into vesicles and delivered to their target site by vesicular fission and fusion. Although much has been learned concerning these mechanisms at donor and acceptor compartments, relatively little attention has been paid to understanding how membrane homeostasis is preserved. This aspect is particularly important in neurosecretory cells in which intense membrane trafficking and mixing occur between the plasma membrane and secretory vesicle membranes during neurotransmission and hormone release.

In neuroendocrine cells, hormones and neuropeptides are stored in large dense-core vesicles (LDCV), the secretory granules. Exocytotic release of hormones and neuropeptides into the blood stream involves four main trafficking steps: i) the tethering of granules to the plasma membrane, ii) their docking at the exocytotic sites through the assembly of SNARE proteins, iii) the priming step rendering the docked granules competent for fusion and iv) the fusion between the granule membrane and the plasma membrane leading to the 


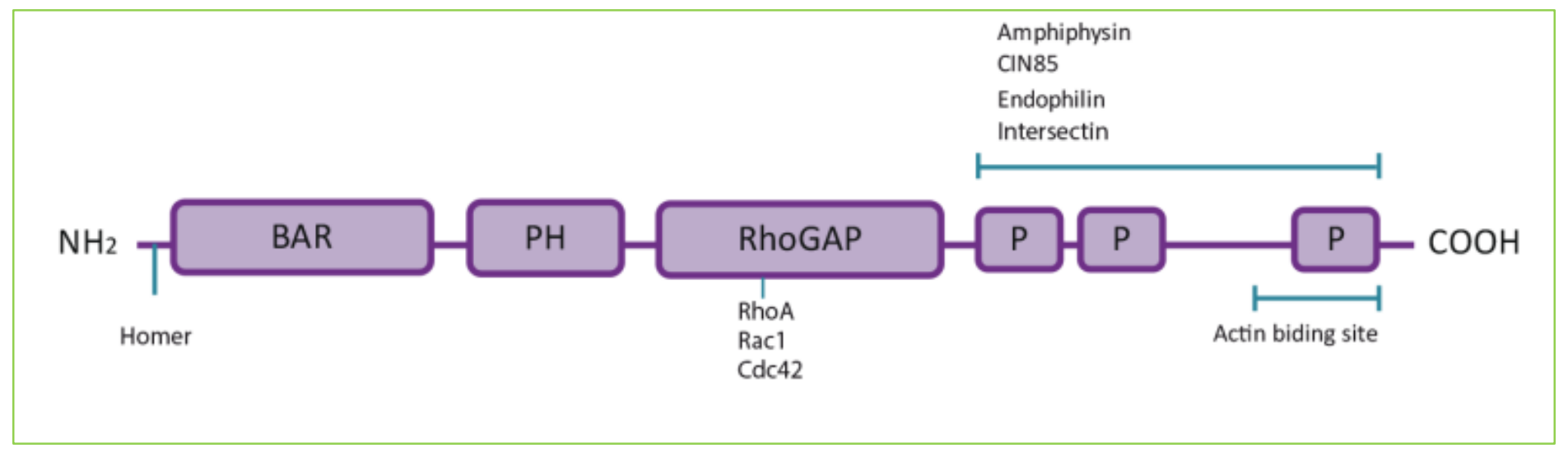

Figure 1. Schematic representation of Oligophrenin-1. OPHN1 is a Rho family GTPase activating protein (Rho-GAP) that contains a $\mathrm{N}$-terminal BAR domain, which senses and binds curved membranes and a Pleckstrin Homology (PH) domain, which binds phosphatidylinositol lipids. The catalytic GAP domain inhibits RhoA, Rac1, and Cdc42 in vitro ${ }^{[2]}$. Moreover, at the C-terminal part, OPHN1 contains an actin-binding site and three Proline-rich sites permitting the interaction with SH3 domain containing proteins including amphiphysinl and II, CIN85, endophilinA1 and B2, Homer1 and intersectin1 [5, 7, 8, 28].

formation of a fusion pore that expands to release the granule content. While the mechanisms underlying exocytosis per se have been extensively characterized in neuroendocrine cells, how the composition, integrity and functionality of the plasma membrane are maintained after exocytosis is obscure. However, in neuroendocrine chromaffin cells from the adrenal gland, we have proposed that secretory granule proteins cluster together at the plasma membrane after full fusion exocytosis before their recapture by compensatory endocytosis ${ }^{[1]}$. One of our current research goals is to determine the molecular machinery that enables this sorting, segregation and recapture of secretory granule membrane components.

The characteristics of oligophrenin-1 (OPHN1, figure 1) attracted our attention and suggested it might be a potential candidate for linking the exocytosis and endocytosis in chromaffin cells. OPHN1 was originally discovered as one of the genes implicated in cognitive dysfunctions ${ }^{[2]}$, and has previously been shown to regulate membrane trafficking events linked to synaptic functions, including plasticity, post-synaptic receptor trafficking, and synaptic vesicle recycling ${ }^{[3-9]}$. It is a multi-domain protein which can interact with membranes through a BAR domain affecting membrane curvature and Pleckstrin Homology $(\mathrm{PH})$ domain which facilitates membrane binding. In addition, OPHN1 is a GTPase activating protein (GAP) of the Rho-family ${ }^{[2]}$ and can also interact directly with cytoskeleton actin filaments and with scaffold proteins with SH3 domains ${ }^{[10]}$. All these particular features prompted us to examine the role of OPHN1 in exo-endocytosis in chromaffin cells.

\section{Oligophrenin-1, a new actor in calcium-regulated exocytosis}

OPHN1 was found to be expressed in adrenal medulla tissue and was localized in the plasma membrane and in the cytoplasm of the adrenal chromaffin cells. Amperometric measurements of catecholamine secretion from cultured chromaffin cells from Ophn1 knock-out mice and from cultured bovine chromaffin cells with knocked down OPHN1 expression revealed that the fusion pore formation is perturbed in the absence of OPHN1. In the bovine chromaffin cells, this phenotype could be rescued by re-expressing OPHN1, but not the GAP-dead OPHN1 mutant $\left(\mathrm{OPHN} 1_{\mathrm{R} 409 \mathrm{~L}}\right)$, indicating that inactivation of a Rho-GTPase by OPHN1 is necessary for fusion pore formation. OPHN1 probably specifically inactivates RhoA during exocytosis because knocking down OPHN1 only significantly increased the level of activated RhoA in stimulated PC12 cells.

Several hypotheses can be proposed to explain how OPHN1 and the RhoA pathways contribute to formation of the fusion pore (figure 2). One possibility is that OPHN1 regulates SNARE complex formation during exocytosis via the RhoA/Rho-kinase (ROCK) pathway. Indeed, deregulation of SNARE complex formation impacts fusion pore formation ${ }^{[11-13]}$. ROCK-induced phosphorylation of the t-SNARE syntaxin-1A favors its interaction with tomosyn, a negative regulator of secretion ${ }^{[14]}$. Therefore, OPHN1 may prevent the interaction between syntaxin-1A and tomosyn by inactivating RhoA/ROCK and thereby enhances the formation of the fusion pore. In neurons which express OPHN1 both pre- and post-synaptically, the role of OPHN1 has been studied. While a direct function of OPHN1 in 


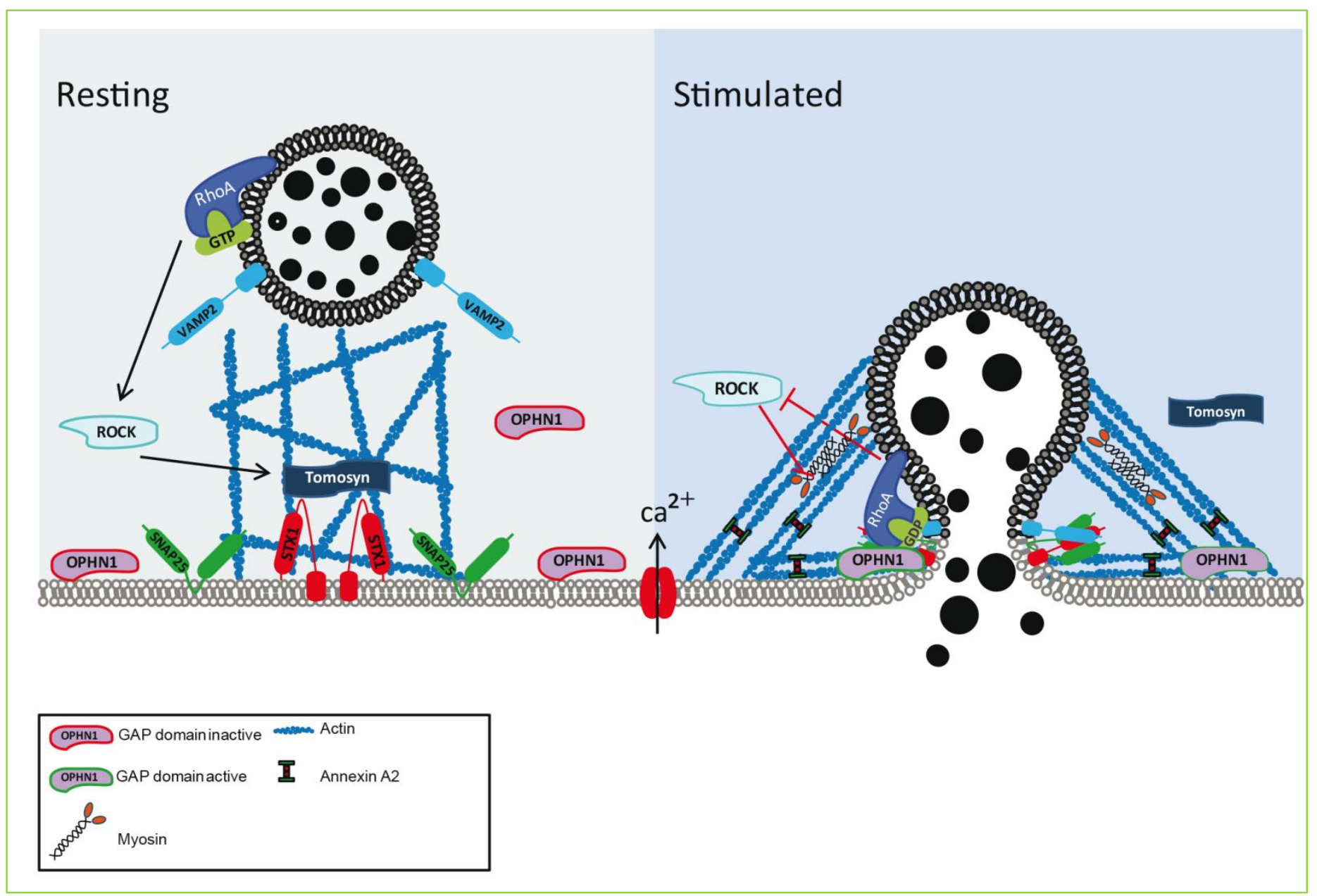

Figure 2. Hypothetical model for the role of OPHN1 in exocytosis in chromaffin cells. In resting condition, the GAP domain of OPHN1 is non-functional which maintains active the granule-bound RhoA. Activated-RhoA might activate its downstream effector, the Rho-kinase (ROCK) that subsequently phosphorylates tomosyn enhancing the interaction between syntaxin-1 and tomosyn and restricting the formation of SNARE complex (VAMP-2/Syntaxin-1/SNAP25). In secretagogue-stimulated chromaffin cells, the rise in cytosolic calcium somehow triggers the activation of the OPHN1 GAP activity and the subsequent inactivation of RhoA. How inactivation of RhoA is linked to fusion pore formation is currently unknown. Inhibition of the RhoA-ROCK pathway might favor the SNARE complex formation by preventing tomosyn/syntaxin-1 interaction. Alternatively, it might regulate the level of myosin light chain (MLC) phosphorylation and therefore modulate the myosin-induced forces required for exocytosis ${ }^{[32]}$. These two hypotheses are not mutually exclusive and required further investigations.

neurotransmitter release has not clearly been explored, two studies suggest that it might be the case. It has been shown in hippocampal neurons from Ophn1 knock-out mice that the size of the readily releasable pool is reduced and that short-term plasticity like paired-pulse facilitation is altered suggesting defects in vesicle availability for exocytosis and the probability of neurotransmitter release ${ }^{[3,9]}$. In our study, the number of released vesicle was slightly reduced in $\mathrm{KO}$ chromaffin cells. However, carbon-fiber amperometry does not provide any indication about the size of the different vesicular pools. To obtain further information about vesicle pool sizes in these cells, capacitance measurements combined with carbon-fiber amperometry are required.

Alternatively, OPHN1 may modulate the remodeling of the actin cytoskeleton which occurs during exocytosis. Not only can OPHN1 interact directly with actin filaments but the RhoA/ROCK pathway is known to regulate the acto-myosin contraction by enhancing myosin light chain phosphorylation either directly or through the activity of the myosin light chain phosphatase $[15,16]$. Accordingly, modulation of the acto-myosin activity is known to affect fusion pore properties [17-19]. Moreover, previous studies from our laboratory demonstrated that inactivation of granule-bound RhoA during exocytosis is linked to the organization of the cortical actin network in chromaffin cells [20-22]. Subsequently, activation of the Rho-GTPase Cdc 42 was shown to enhance de novo polymerization of actin filaments at granule docking sites ${ }^{[23]}$ and annexin-A2 has recently been reported to bundle actin filaments in order to connect the granule membrane with the plasma membrane and regulate the fusion pore dynamics ${ }^{[24]}$. It is tempting to propose that OPHN1-induced 
inhibition of RhoA is somehow required in this sequence of molecular events. For example, the interaction of OPHN1 with F-actin might allow the specific recruitment of OPHN1 at docking sites in order to inactivate granule-bound RhoA. This inactivation of RhoA could in turn prevent myosin light chain phosphorylation by ROCK, thus modifying the forces generated by the annexin-A2-induced bundles of filament at the interface between the granule and plasma membranes and affect fusion pore properties.

\section{Oligophrenin-1 regulates compensatory endocytosis of secretory granules}

Using an assay developed in our laboratory to specifically follow the granule membrane recapture after exocytosis (for more details see ${ }^{[1]}$ ), we observed a severe decrease (around $70 \%$ ) of the compensatory endocytosis in chromaffin cells cultured from Ophn1 KO mice. This reveals a major role of OPHN1 in the pathway mediating compensatory endocytosis of the secretory granule membrane. We then demonstrated that overexpression of OPHN1 mutant lacking the BAR domain reproduced the inhibitory effect on granule membrane recapture in bovine chromaffin cells whereas the GAP-dead OPHN1 $1_{\mathrm{R} 409 \mathrm{~L}}$ mutant had no effect. These data indicate that the BAR domain of OPHN1 is essential for compensatory endocytosis in chromaffin cells.

The role of OPHN1 in secretory granule recapture is easier to correlate with the data obtained in neurons. OPHN1 is pivotal in neurons for synaptic vesicle recycling or post-synaptic receptor endocytosis ${ }^{[5,7,25]}$, yet surprisingly, the potential implication of the BAR domain of OPHN1 in these processes has never been questioned. On the contrary, it has been shown that OPHN1 is able to recruit and interact with other BAR domain-containing proteins involved in endocytosis: endophilinA1, endophilinB2 and amphiphysin $[5,7]$. Moreover, an interaction between OPHN1 and endophilinA1 has been proposed to be essential for synaptic vesicle recycling ${ }^{[7]}$. This clearly raises an issue regarding the exact function of the BAR domain of OPHN1 at synapses. For the moment, the mechanisms by which OPHN1 regulates compensatory endocytosis in chromaffin cells are open to speculation.

In order to bind and stabilize precise membrane curvature, BAR domains need to form homo- or hetero-dimers leading to the formation of a banana shape. Since the interaction with other BAR-domain containing proteins occurs through the C-terminal proline-rich domain of OPHN1, formation of hetero-dimers of BAR domains is a possibility. Whether such a conformation occurs in neurons and/or chromaffin cells requires further investigation. OPHN1 could also act as a scaffold protein by recruiting other functional proteins at the place where membranes are curved; in this case where the vesicle will be endocytosed [26, 27]. For example, we have previously shown that OPHN1 can interact with intersectin 1 (ITSN1) in neuroendocrine cells, a key regulator of endocytosis ${ }^{[28]}$.

\section{Conclusion: Oligophrenin-1 is a molecular switch} between exocytosis and endocytosis of secretory granules

Altogether, these results clearly demonstrate a role for OPHN1 in the molecular machinery underlying neuroendocrine secretion. In particular, OPHN1 has a bifunctional role both in calcium-regulated exocytosis and compensatory endocytosis. However, an important remaining question is how does OPHN1 switch from its GAP activity required for exocytosis to its BAR activity required for compensatory endocytosis? Interestingly, the BAR domain itself can interact with the GAP domain leading to the inhibition of the GAP domain ${ }^{[10,29]}$. In addition, interaction between the GAP and BAR domains seems to potentiate the BAR-mediated ability of membrane binding [29]. An attractive scenario can therefore be considered. OPHN1 is recruited to the exocytotic site in an "open conformation" and regulates fusion pore formation through its Rho-GAP activity. Once the intra-granular contents are released, OPHN1 shifts to an auto-inhibited conformation which blocks the GAP activity and enhances the activity of the BAR domain required for endocytosis. How the BAR domain binds to the GAP domain and how the switch from one conformation to the other is regulated requires further investigations.

To conclude, OPHN1 functions as a structural checkpoint that spatially and temporally couples exocytosis and endocytosis in neuroendocrine cells, and appears as a good candidate to ensure a fine tuning of hormone secretory activity. The next challenges will be to decipher the mechanisms by which OPHN1 regulates fusion pore dynamics and to further explore the importance of the OPHN1 BAR domain in endocytic processes. Genetic mutations in OPHN1 gene leading either to the deletion of the BAR domain or to a non-functional BAR domain have recently been reported in patient with an intellectual disability ${ }^{[30,31]}$. Along this same line, it would be of primary interest to investigate whether, patients with mutations in the OPHN1 gene display neuroendocrine disorders in addition to neuronal defects and associated cognitive disabilities.

\section{Conflicting interests}

The authors declare that there is no conflict of interest. 
http://www.smartscitech.com/index.php/nc

\section{Acknowledgement}

We thank Nancy Grant and Sylvette Chasserot-Golaz for the critical reading of the manuscript and fruitful discussion. The work discussed in this article has been supported by grants from "Ligue Contre le Cancer" (comité Alsace 2012-13) to SG and by a Ph.D fellowship from "Région Alsace" and "Ligue Contre le Cancer" to SH and a Ph.D fellowship from CONICYT Chile to CEA.

\section{References}

1. Ceridono M, Ory S, Momboisse F, Chasserot-Golaz S, Houy S, Calco V, et al. Selective recapture of secretory granule components after full collapse exocytosis in neuroendocrine chromaffin cells. Traffic 2011; 12: 72-88.

2. Billuart P, Bienvenu T, Ronce N, des Portes V, Vinet MC, Zemni $\mathrm{R}$, et al. Oligophrenin-1 encodes a rhoGAP protein involved in X-linked mental retardation. Nature 1998; 392: 923-926.

3. Khelfaoui M, Denis C, van Galen E, de Bock F, Schmitt A, Houbron $\mathrm{C}$, et al. Loss of X-linked mental retardation gene oligophrenin1 in mice impairs spatial memory and leads to ventricular enlargement and dendritic spine immaturity. J Neurosci 2007; 27: 9439-9450.

4. Khelfaoui M, Gambino F, Houbaert X, Ragazzon B, Muller C, Carta M, et al. Lack of the presynaptic RhoGAP protein oligophrenin1 leads to cognitive disabilities through dysregulation of the cAMP/PKA signalling pathway. Philos Trans R Soc Lond B Biol Sci 2014; 369: 20130160.

5. Khelfaoui M, Pavlowsky A, Powell AD, Valnegri P, Cheong KW, Blandin $\mathrm{Y}$, et al. Inhibition of RhoA pathway rescues the endocytosis defects in Oligophrenin 1 mouse model of mental retardation. Hum Mol Genet 2009; 18: 2575-2583.

6. Nadif Kasri N, Nakano-Kobayashi A, Malinow R, Li B and Van Aelst L. The Rho-linked mental retardation protein oligophrenin-1 controls synapse maturation and plasticity by stabilizing AMPA receptors. Genes Dev 2009; 23: 1289-1302.

7. Nakano-Kobayashi A, Kasri NN, Newey SE and Van Aelst L. The Rho-linked mental retardation protein OPHN1 controls synaptic vesicle endocytosis via endophilin A1. Curr Biol 2009; 19: 1133-1139.

8. Nakano-Kobayashi A, Tai Y, Nadif Kasri N and Van Aelst L. The X-linked mental retardation protein OPHN1 interacts with Homer1b/c to control spine endocytic zone positioning and expression of synaptic potentiation. J Neurosci 2014; 34: 8665-8671.

9. Powell AD, Gill KK, Saintot PP, Jiruska P, Chelly J, Billuart P, et al. Rapid reversal of impaired inhibitory and excitatory transmission but not spine dysgenesis in a mouse model of mental retardation. J Physiol 2012; 590: 763-776.

10. Fauchereau F, Herbrand U, Chafey P, Eberth A, Koulakoff A, Vinet MC, et al. The RhoGAP activity of OPHN1, a new F-actin-binding protein, is negatively controlled by its amino-terminal domain. Mol Cell Neurosci 2003; 23: 574-586.

11. Bretou M, Anne C and Darchen F. A fast mode of membrane fusion dependent on tight SNARE zippering. J Neurosci 2008; 28 : 8470-8476.
12. Chang CW, Hui E, Bai J, Bruns D, Chapman ER and Jackson MB. A structural role for the synaptobrevin 2 transmembrane domain in dense-core vesicle fusion pores. J Neurosci 2015; 35: 5772-5780.

13. Fang Q, Zhao Y, Herbst AD, Kim BN and Lindau M. Positively charged amino acids at the SNAP-25 C terminus determine fusion rates, fusion pore properties, and energetics of tight SNARE complex zippering. J Neurosci 2015; 35: 3230-3239.

14. Sakisaka T, Baba T, Tanaka S, Izumi G, Yasumi M and Takai Y. Regulation of SNAREs by tomosyn and ROCK: implication in extension and retraction of neurites. J Cell Biol 2004; 166: 17-25.

15. Amano M, Ito M, Kimura K, Fukata Y, Chihara K, Nakano T, et al. Phosphorylation and activation of myosin by Rho-associated kinase (Rho-kinase). J Biol Chem 1996; 271: 20246-20249.

16. Totsukawa G, Yamakita Y, Yamashiro S, Hartshorne DJ, Sasaki Y and Matsumura F. Distinct roles of ROCK (Rho-kinase) and MLCK in spatial regulation of MLC phosphorylation for assembly of stress fibers and focal adhesions in $3 \mathrm{~T} 3$ fibroblasts. J Cell Biol 2000; 150: 797-806.

17. Berberian K, Torres AJ, Fang Q, Kisler K and Lindau M. F-actin and myosin II accelerate catecholamine release from chromaffin granules. J Neurosci 2009; 29: 863-870.

18. Doreian BW, Fulop TG and Smith CB. Myosin II activation and actin reorganization regulate the mode of quantal exocytosis in mouse adrenal chromaffin cells. J Neurosci 2008; 28: 4470-4478.

19. Neco P, Fernandez-Peruchena C, Navas S, Gutierrez LM, de Toledo GA and Ales E. Myosin II contributes to fusion pore expansion during exocytosis. J Biol Chem 2008; 283: 10949-10957.

20. Bader MF, Doussau F, Chasserot-Golaz S, Vitale N and Gasman S. Coupling actin and membrane dynamics during calcium-regulated exocytosis: a role for Rho and ARF GTPases. Biochim Biophys Acta 2004; 1742: 37-49.

21. Gasman S, Chasserot-Golaz S, Hubert P, Aunis D and Bader MF. Identification of a potential effector pathway for the trimeric Go protein associated with secretory granules. Go stimulates a granule-bound phosphatidylinositol 4-kinase by activating RhoA in chromaffin cells. J Biol Chem 1998; 273: 16913-16920.

22. Gasman S, Chasserot-Golaz S, Popoff MR, Aunis D and Bader MF. Trimeric G proteins control exocytosis in chromaffin cells. Go regulates the peripheral actin network and catecholamine secretion by a mechanism involving the small GTP-binding protein Rho. J Biol Chem 1997; 272: 20564-20571.

23. Gasman S, Chasserot-Golaz S, Malacombe M, Way M and Bader MF. Regulated exocytosis in neuroendocrine cells: a role for subplasmalemmal Cdc42/N-WASP-induced actin filaments. Mol Biol Cell 2004; 15: 520-531.

24. Gabel M, Delavoie F, Demais V, Royer C, Bailly Y, Vitale N, et al. Annexin A2-dependent actin bundling promotes secretory granule docking to the plasma membrane and exocytosis. J Cell Biol 2015; 210: 785-800.

25. Nadif Kasri N, Nakano-Kobayashi A and Van Aelst L. Rapid synthesis of the X-linked mental retardation protein OPHN1 mediates mGluR-dependent LTD through interaction with the endocytic machinery. Neuron 2011; 72: 300-315.

26. Daumke O, Roux A and Haucke V. BAR domain scaffolds in dynamin-mediated membrane fission. Cell 2014; 156: 882-892. 
27. Mim C and Unger VM. Membrane curvature and its generation by BAR proteins. Trends Biochem Sci 2012; 37: 526-533.

28. Gubar O, Houy S, Billuart P, Kropyvko S, Tsyba L, Gasman S, et al. GTPase-activating protein oligophrenin 1 is a new partner of multifunctional adapter protein intersectin 1. Biopolymers and Cell. 2012; 28: 357-362.

29. Eberth A, Lundmark R, Gremer L, Dvorsky R, Koessmeier KT, McMahon HT, et al. A BAR domain-mediated autoinhibitory mechanism for RhoGAPs of the GRAF family. Biochem J 2009; 417: 371-377.

30. Pirozzi F, Di Raimo FR, Zanni G, Bertini E, Billuart P, Tartaglione $\mathrm{T}$, et al. Insertion of 16 amino acids in the BAR domain of the oligophrenin 1 protein causes mental retardation and cerebellar hypoplasia in an Italian family. Hum Mutat 2011; 32: E2294-2307.

31. Santos-Reboucas CB, Belet S, Guedes de Almeida L, Ribeiro MG, Medina-Acosta E, Bahia PR, et al. A novel in-frame deletion affecting the BAR domain of OPHN1 in a family with intellectual disability and hippocampal alterations. Eur J Hum Genet 2014; 22: 644-651.

32. Papadopulos A, Gomez GA, Martin S, Jackson J, Gormal RS, Keating DJ, et al. Activity-driven relaxation of the cortical actomyosin II network synchronizes Munc18-1-dependent neurosecretory vesicle docking. Nat Commun 2015; 6: 6297. 